\title{
Association between susceptibility to photodynamic oxidation and the genetic background of Staphylococcus aureus
}

\author{
A. Rapacka-Zdonczyk • A. Rhod Larsen • J. Empel • \\ A. Patel • M. Grinholc
}

Received: 30 July 2013 / Accepted: 18 September 2013 /Published online: 26 October 2013

(C) The Author(s) 2013. This article is published with open access at Springerlink.com

\begin{abstract}
Multidrug resistant strains of Staphylococcus aureus are a major cause of skin and soft tissue infections requiring the development of novel and alternative therapeutic options. Photodynamic oxidation is the cornerstone of antimicrobial photodynamic therapy (aPDT) involving the combined use of light and a photosensitizer, which, in the presence of oxygen, originates cytotoxic species capable of oxidizing biological molecules and leads to inactivation of target cells. We have previously shown that susceptibility to aPDT differs significantly across $S$. aureus isolates and could be associated with several genetic elements. However, the effect of the photodynamic process regarding the $S$. aureus genetic background has never been reported. We have compared the genetic backgrounds of the strains (SCCmec types, spa types and main clonal complexes) with respect to their susceptibility to protoporphyrin IX-mediated photodynamic inactivation. SCCmec typing revealed no differences in response to photoinactivation. However, detection of spa types and clonal complexes clustered the studied population of MRSA strains
\end{abstract}

Electronic supplementary material The online version of this article (doi:10.1007/s10096-013-1987-5) contains supplementary material, which is available to authorized users

A. Rapacka-Zdonczyk $\cdot$ M. Grinholc $(\triangle)$

Laboratory of Molecular Diagnostics, Department of Biotechnology, Intercollegiate Faculty of Biotechnology, University of Gdansk and Medical University of Gdansk, Kladki 24, 80-822 Gdansk, Poland e-mail: mariusz.grinholc@biotech.ug.edu.pl

A. R. Larsen

Staphylococcus Laboratory, Statens Serum Institute,

2300 Copenhagen, Denmark

J. Empel

Department of Molecular Microbiology, National Medicines Institute, Warsaw, Poland

A. Patel

Centre for Clinical Infection and Diagnostics Research, Department of Infectious Diseases, St Thomas' Hospital, London, UK according to their response to photodynamic oxidation. Clonal complex 1 (CC1) accounted for elevated resistance and CC30 (ST36) for susceptibility to photoinactivation. Moreover, spa typing identified isolates resistant ( $\mathrm{t} 032)$ and susceptible to photodynamic oxidation ( $\mathrm{t} 051, \mathrm{t} 015)$. The very tight association between clonal lineages and response to photodynamic inactivation indicates the important role of genetic background for aPDT efficacy. These results make a case for the development of a diagnostic tool with the predictive value of aPDT efficacy according to an identified genetic background of $S$. aureus isolates.

\section{Introduction}

Healthcare-associated infections, especially those caused by methicillin-resistant Staphylococcus aureus strains (HAMRSA), are a great danger to both hospitalized and immunocompromised patients, in whom the organism causes high morbidity and mortality [1]. S. aureus displays extensive genetic variability. Genotyping analyses, such as multilocus sequence typing (MLST) and protein A (spa) sequence typing, demonstrated that the $S$. aureus population structure is highly clonal with six major clonal complexes (CCs) $(\mathrm{CC} 1, \mathrm{CC} 5, \mathrm{CC} 8, \mathrm{CC} 22$, CC30, and CC45) [2-4]. Most mobile genetic elements can readily spread horizontally among $S$. aureus strains of the same clonal cluster, while transfer between clusters is limited. $S$. aureus strains are generally classified into two classes according to their resistance to $\beta$-lactam antibiotics: methicillin-sensitive $S$. aureus (MSSA) and MRSA strains. One of the resistance mechanisms to $\beta$-lactams used by MRSA is based on the production of an alternative penicillin-binding protein (PBP) encoded by the $m e c A$ gene; this protein is termed PBP2a. In MRSA, exposure to $\beta$-lactams inactivates the four high-binding-affinity PBPs normally present. PBP2a, which displays a low affinity for $\beta$ lactams, takes over the functions of these PBPs, permitting the cell to grow. The mecA gene is located on a mobile genetic 
element integrated into the bacterial chromosome and called the staphylococcal cassette chromosome mec (SCCmec) [5]. Eleven different types of $\mathrm{SCCmec}$, assigned SCCmec I to SCCmec XI, have been defined so far. Most epidemiological investigations focus on types I to VI, and studies on SCCmec types VII to XI are rarely reported [6-9]. This genetic element is widely used for the determination of MRSA clones. Identification of SCCmec type can be used to predict strain origin, as the majority of nosocomial isolates harbor SCCmec I, II, or III, whereas community-associated MRSA strains (CA-MRSA) are characterized by SCCmec IV and V [8-10]. Furthermore, it seems that $\mathrm{SCCmec}$ element is involved in staphylococcal virulence. It has been shown that $\mathrm{SCCmec}$ type-associated virulence factors substantially influence the progression of staphylococcal bacteremia, and SCCmec II $S$. aureus strains cause the highest morbidity among hospitalized patients [11]. Thus, the determination of SCCmec types, clonal complexes, or spa types may have an important diagnostic value. Whilst no single typing method meets all the requirements for typing techniques, there have been recent trends toward the use of spa, MLST, and SCCmec methods [12]. Large typing databases and networks have been developed to handle the data. The spa typing method is based on sequencing of the polymorphic $\mathrm{X}$ region of the protein $\mathrm{A}$ gene (spa), present in all strains of S. aureus. The X region consists of a variable number of 24-bp repeats flanked by well-conserved regions. This single-locus sequence-based typing method combines a number of technical advantages, such as rapidity, reproducibility, and portability. Moreover, because of its repeat structure, the spa locus simultaneously indexes micro- and macrovariations, enabling the use of spa typing in both local and global epidemiological studies [13]. The current terminology to describe a lineage is based on multilocus sequence typing (MLST) clonal complexes (CCs). MLST involves sequencing seven housekeeping genes and assigning a sequence type (ST) number to isolates that are identical [12]. MLST is recommended for use in regional laboratories and is a useful tool for defining lineages and scientific analysis. These methods, plus SCCmec, provide the minimum requirements for appropriate molecular epidemiology [12].

The antimicrobial photodynamic therapy (aPDT) has gained increasing importance because of its ability to eradicate a broad spectrum of pathogenic microorganisms [14, 15]. In the clinical area it could be a potential alternative to the antibiotics used to treat staphylococcal infections. The photodynamic process involves the combined use of light, molecular oxygen, and a photosensitive compound (photosensitizer) [16]. This interaction leads to production of reactive oxygen species and free radicals responsible for oxidation of many biological molecules (enzymes, proteins, lipids, and nucleic acids), leading to cell death [17]. Additionally, the resistance mechanisms towards photodynamic inactivation have not yet been observed [18, 19]. However, our studies of the photoinactivation (PDI) of multiresistant pathogenic bacteria have shown that the effectiveness of PDI is strain-dependent and that one should search for possible strategies to overcome resistant phenotype, but, on other hand, to develop the diagnostic tool clustering strains according to their susceptibility to PDI [20-24]. The mechanism underlying this phenomenon is still poorly understood. Thus, by applying SCCmec typing and collecting the isolates representing different spa genotypes and clonal clusters, we aimed, in particular, to assess the contribution of the bacterial genetic background in $S$. aureus response to photodynamic oxidation.

\section{Materials and methods}

Bacterial isolates

In total, $438 S$. aureus strains (418 MRSA and 20 MSSA) were used. Of them, 321 clinical MRSA strains were isolated between 2002 and 2012 at the Provincial Hospital in Gdansk and Hospital in Koszalin (Poland) and were previously characterized. The isolates were characterized by Gram staining and the ability to produce coagulase and the clumping factor using Slidex Staph Plus (bioMèrieux, France) and were reidentified to the species level using the MALDI-TOF MS (Bruker Daltonics, Germany). Methicillin resistance was determined using a disc-diffusion method as well as a latex test detecting PBP2a protein (Staphytect Plus; Oxoid, Lenexa,KS, USA) [25]. Of the 321 strains collected, $40.5 \%$ (130 isolates) were isolated from surveillance cultures, $46.4 \%$ (149 isolates) from patients with local infections, $11.8 \%$ (38 isolates) from patients with bacteremia and generalized infections and $1.3 \%$ (4 isolates) from infections connected with endoprostheses. An additional 97 MRSA and 20 MSSA isolates, with identified CCs and spa type were obtained through the Network of Antimicrobial Resistance in Staphylococcus aureus (NARSA), Staphylococcus Laboratory, Statens Serum Institute in Denmark, Centre for Clinical Infection and Diagnostics Research in the UK, University of Oviedo in Spain, and National Medicines Institute in Poland. The strains are listed in Online Resource 1. Clonal complexes (CC) were determined with the use of MLST. All clonal complexes, excluding CC8, were represented by single ST. CC8 included strains with ST8 and ST247. Additionally, the reference strains of $S$. aureus listed in Table 1 were used to optimize SCCmec type determination. The abundance of $S$. aureus isolates in each group is provided in Table 2. The molecular characteristics of isolates are provided in Table 3.

\section{MALDI-TOF MS}

Three hundred and twenty-one $S$. aureus clinical strains isolated from hospitals in Poland were examined by MALDITOF MS using a Microflex LT instrument (Bruker Daltonics, 
Table 1 Primers and reference strains used in the study

\begin{tabular}{|c|c|c|c|c|}
\hline Strain used as standard & Primer & Oligonucleotide sequence $\left(5^{\prime}-3^{\prime}\right)$ & Size (bp) & Reference \\
\hline NCTC 10442 & SCC I & $\begin{array}{l}\text { Forward: GCT TTA AAG AGT GTC GTT ACA GG } \\
\text { Reverse: GTT CTC TCA TAG TAT GAC GTC C }\end{array}$ & 613 & {$[27]$} \\
\hline $\mathrm{N} 315$ & SCC II & $\begin{array}{l}\text { Forward: GAT TAC TTC AGA ACC AGG TCA T } \\
\text { Reverse: TAA ACT GTG TCA CAC GAT CCA T }\end{array}$ & 287 & {$[27]$} \\
\hline $85 / 2082$ & SCC III & $\begin{array}{l}\text { Forward: CAT TTG TGA AAC ACA GTA CG } \\
\text { Reverse: GTT ATT GAG ACT CCT AAA GC }\end{array}$ & 243 & [27] \\
\hline $8 / 6-3 \mathrm{P}$ & $\mathrm{SCC} I V b$ & $\begin{array}{l}\text { Forward: AGT ACA TTT TAT CTT TGC GTA } \\
\text { Reverse: AGT CAT CTT CAA TAT GGA GAA AGT A }\end{array}$ & 1,000 & {$[27]$} \\
\hline $81-108$ & SCC IVc & $\begin{array}{l}\text { Forward: TCT ATT CAA TCG TTC TCG TAT T } \\
\text { Reverse: TCG TTG TCA TTT AAT TCT GAA CT }\end{array}$ & 677 & {$[27]$} \\
\hline JSCS 4469 & SCC IVd & $\begin{array}{l}\text { Forward: AAT TCA CCC GTA CCT GAG AA } \\
\text { Reverse: AGA ATG TGG TTA TAA GAT AGC TA }\end{array}$ & 1,242 & {$[27]$} \\
\hline WIS & SCC V & $\begin{array}{l}\text { Forward: GAA CAT TGT TAC TTA AAT GAG CG } \\
\text { Reverse: TGA AAG TTG TAC CCT TGA CAC C }\end{array}$ & 325 & {$[27]$} \\
\hline NCTC 10442 & $\mathrm{Sa} 442$ & $\begin{array}{l}\text { Forward: AAT CTT TGT CGG TAC ACG ATA TTC TTC ACG } \\
\text { Reverse: CGT AAT GAG ATT TCA GTA GAT AAT ACA ACA }\end{array}$ & 108 & {$[27]$} \\
\hline NCTC 10442 & mecA & $\begin{array}{l}\text { Forward: TCC AGA TTA CAA CTT CAC CAG G } \\
\text { Reverse: CCA CTT CAT ATC TTG TAA CG }\end{array}$ & 162 & {$[27]$} \\
\hline
\end{tabular}

Leipzig, Germany), Flexcontrol 3.0 software and the Biotyper 2.0 database (Bruker Daltonics, Germany). The data analysis was performed according to the manufacturer's instructions. The samples were covered with $1 \mathrm{ml}$ matrix solution (a

Table 2 Distribution of methicillin-resistant Staphylococcus aureus (MRSA) isolates by typing procedures

${ }^{a}$ Only types represented by more than three isolates were included (all types are listed in Online Resource 1)

\begin{tabular}{ll}
\hline $\begin{array}{l}\text { Typing } \\
\text { procedures }\end{array}$ & $\begin{array}{l}\text { Number }(\%) \text { of } \\
\text { isolates }\end{array}$ \\
\hline Clonal complex \\
CC1 & $5(5.15)$ \\
CC5 & $22(22.65)$ \\
CC8 & $25(25.80)$ \\
CC22 & $8(8.25)$ \\
CC30 & $3(3.10)$ \\
CC45 & $34(35.05)$ \\
SCCmec & \\
I & $31(7.40)$ \\
II & $222(53.10)$ \\
III & $22(5.25)$ \\
IV & $124(29.70)$ \\
V & $19(4.55)$ \\
spa type & \\
t002 & $15(15.50)$ \\
t008 & $7(7.20)$ \\
t015 & $12(12.40)$ \\
t026 & $4(4.10)$ \\
t032 & $6(6.20)$ \\
t051 & $6(6.20)$ \\
t064 & $6(6.20)$ \\
t230 & $4(4.10)$ \\
Other & $37(38.10)$ \\
\hline &
\end{tabular}

saturated solution of a-cyano-4-hydroxycinnamic acid in $50 \%$ acetonitrile, $2.5 \%$ trifluoroacetic acid). The analyzed mass range of spectra was 2,000-20,000 m/z. Each spectrum was obtained after 240 shots in an automatic acquisition mode. For the identification approach, a mass-to-charge range of 3,000-15,000 Da was used. Identification was performed in duplicate and the higher score was retained. The identification score cut-off values were applied on each measurement according to the manufacturer's instructions. According to this score system, a score of $<2$ is recommended for a probable species and a score of greater than 2.3 is recommended for a secure species identification [26].

Nucleic acid isolates

For DNA isolation, strains were grown overnight in nutrient trypticase soy broth (TSB) (bioMèrieux, France) at $37^{\circ} \mathrm{C}$ for $24 \mathrm{~h}$. Then, $1.5 \mathrm{ml}$ of the bacterial culture was centrifuged at $5000 \mathrm{x} \mathrm{g}$ for $10 \mathrm{~min}$. The pellet was suspended in $180 \mu \mathrm{l}$ of lysis buffer (20 mM Tris-HCl, pH 8.0, 2 mM EDTA, $1.2 \%$ Triton X-100, lysozyme $20 \mathrm{mg} / \mathrm{ml}$ ) and the GeneJET ${ }^{\mathrm{TM}} \mathrm{Ge}-$ nomic DNA Purification Kit (Thermo Scientific, Vilnius, Lithuania) was used for DNA isolation according to the manufacturer's procedure. The DNA concentrations were measured using NanoDrop ND-1000 (Thermo Scientific) and were within the range 10 to $100 \mathrm{ng} / \mu \mathrm{l}$. In addition, the quality of the isolated DNA was confirmed by gel electrophoresis.

SCCmec type-specific multiplex PCR

A two-tube multiplex PCR method was performed to identify the SCCmec types from the MRSA isolates [27]. One tube 
Table 3 Molecular characteristics of MRSA isolates

\begin{tabular}{llll}
\hline \multicolumn{2}{l}{ Typing procedure (No. of isolates) } & & \\
\hline CC & ST & SCCmec type & spa type \\
CC1 (5) & ST1 (5) & IV (5) & t125 (1), t128 (2), t175 (2) \\
CC5 (22) & ST5 (22) & II (21) IV(1) & t002 (15), t010 (2), t062 (2) \\
& & & $\mathrm{t} 088(1), \mathrm{t} 242(1), \mathrm{t} 601(1)$ \\
CC8 (16) & ST8 (16) & I (3) II (1) IV (12) & $\mathrm{t} 008(7), \mathrm{t} 024(1), \mathrm{t} 064(6)$ \\
& & & $\mathrm{t} 2238(1), \mathrm{t} 451(1)$ \\
CC22 (8) & ST247 (9) & I (5) IV (4) & $\mathrm{t} 051(6), \mathrm{t} 052(2), \mathrm{t} 770(1)$ \\
CC30 (3) & ST36 (3) & IV (8) & $\mathrm{t} 005(1), \mathrm{t} 032(6), \mathrm{t} 852(1)$ \\
CC45 (34) & ST45 (34) & II (1) III (1) IV (31) V (1) & $\mathrm{t} 018(2), \mathrm{t} 253(1)$ \\
& & & $\mathrm{t} 015(12), \mathrm{t} 026(4), \mathrm{t} 040(1)$ \\
& & & $\mathrm{t} 050(3), \mathrm{t} 065(1), \mathrm{t} 1078(1)$ \\
& & $\mathrm{t} 1081(1), \mathrm{t} 1574(1), \mathrm{t} 230(4)$ \\
ND & ND & I (21) II (203) III (20) IV (61) V (16) & $\mathrm{t} 2135(1), \mathrm{t} 330(1), \mathrm{t} 350(1)$ \\
97 & 97 & 418 & $\mathrm{t} 445(1), \mathrm{t} 583(1), \mathrm{t} 620(1)$ \\
\hline
\end{tabular}

ND not determined

was used to determine SCCmec types I, II, III, and V, and one tube was used to determine SCCmec types IVa, IVb, IVc, IVd, and IVh. In the first reaction, the $S a 442$ and mecA genes were also amplified as a control. $S a 442$ is a gene specific to $S$. aureus, and mecA encodes the alternate penicillin-binding protein responsible for beta-lactam resistance. The two-tube reactions were performed in a $25-\mu$ l reaction mixture containing $4 \mu \mathrm{l}$ of the purified nucleic acid solution, $1 \mathrm{U}$ of DyNAzyme ${ }^{\mathrm{TM}}$ EXT Taq DNA Polymerase (Thermo Scientific), 10X DyNAzyme ${ }^{\mathrm{TM}}$ EXT Buffer (Thermo Scientific), $200 \mu \mathrm{M}$ dNTPs (MetaBion, Martinsried, Germany), and $\mathrm{SCC}$ ec group-specific primers. For the first tube reactions, a $2-\mu \mathrm{M}$ concentration of SCC I primers were used along with a $0.4 \mu \mathrm{M}$ concentration of the primers for $m e c A, S a 442, \mathrm{SCC}$ II, SCC III, and SCC V (primers listed in Table 1). For the second tube reactions, $2 \mu \mathrm{M}$ concentrations of the primers for SCC IV were used (primers listed in Table 1). Amplifications were performed in an Applied Biosystems thermocycler (GeneAmp ${ }^{\circledR}$ PCR System 9700) using the following temperature program: 1 cycle of $4 \mathrm{~min}$ at $95^{\circ} \mathrm{C} ; 30$ cycles of $30 \mathrm{~s}$ at $94{ }^{\circ} \mathrm{C}$, $1 \mathrm{~min}$ and $30 \mathrm{~s}$ at $57^{\circ} \mathrm{C}, 1 \mathrm{~min}$ and $30 \mathrm{~s}$ at $72^{\circ} \mathrm{C}$, and a final cycle of $10 \mathrm{~min}$ at $72^{\circ} \mathrm{C}$. Amplification products were electrophoresed in a $1.8 \%$ agarose gel (BIORON, Ludwigshafen, Germany) containing ethidium bromide and visualized by transillumination (Gel Doc 2000; Bio-Rad) under UV irradiation [27].

\section{Photosynthesizer}

Protoporphyrin IX (PPIX) (MP Biomedicals, LLC, USA) was used as a sensitizer. PPIX was dissolved in dimethyl sulfoxide (DMSO; Sigma, Germany) to make a 1-mM stock solution and was stored in the dark at $-20^{\circ} \mathrm{C}$ until use. The concentration of the photosensitizer was determined spectrophotometrically (extinction coefficient $1.6 \times 10^{5} \mathrm{M}^{-1} \mathrm{~cm}^{-1}$, wavelength $408 \mathrm{~nm}$ ).

Light source

Illumination was performed using a Q.Light ${ }^{\circledR}$ PDT Lamp (b $\& \mathrm{p}^{\circledR}$ Schweiz AG, St Gallen, Switzerland) (ISO 9001 and EN 46001-CE 1275; power of lamp $80 \mathrm{~mW}$; fluence rate $102 \mathrm{~mW} / \mathrm{cm}^{2}$; fluence $6.12 \mathrm{~J} / \mathrm{cm}^{2}$ per minute). The delivered light energy was determined with the use of a light power meter (model LM1; Carl Zeiss, Oberkochen, Germany). The Q.Light ${ }^{\circledR}$ PDT Lamp emits polarized light (polarization level: $98 \%$ ) over a wavelength range of 620 to $780 \mathrm{~nm}$.

PDI studies

Three hundred and twenty-one isolates were already characterized regarding their response to photodynamic inactivation. One hundred and seventeen newly collected $S$. aureus strains (97 MRSA and 20 MSSA isolates) were subjected to photodynamic inactivation (PDI). Bacterial cultures were grown overnight $(24 \mathrm{~h})$ at $37{ }^{\circ} \mathrm{C}$ in nutrient trypticase soy broth (bioMèrieux, Craponne, France) and then diluted with fresh broth to an appropriate density $\left(10^{7} / \mathrm{ml}\right.$ bacterial cells) based on densitometry (Densi Meter II, EMO). Diluted $S$. aureus cultures were incubated with $25 \mu \mathrm{M}$ protoporphyrin IX in the dark at $37{ }^{\circ} \mathrm{C}$ for $30 \mathrm{~min}$. The cells were then transferred to a 96-well microtiter plate $(100 \mu$ per well $)$ and illuminated with appropriate light $\left(50 \mathrm{~J} / \mathrm{cm}^{2}\right)$ at room temperature for $8 \mathrm{~min}$ and $10 \mathrm{~s}$. Following illumination, $10 \mu \mathrm{l}$ aliquots were used to 
determine the colony forming units (CFU). The contents of the wells were mixed before sampling, and the aliquots were serially diluted 10 -fold in PBS $(0.13 \mathrm{mM} \mathrm{NaCl}, 8.1 \mathrm{mM}$ $\mathrm{Na}_{2} \mathrm{HPO}_{4}, 2.68 \mathrm{mM} \mathrm{KCl}, 1.47 \mathrm{mM} \mathrm{KH}_{2} \mathrm{PO}_{4}$ ) to achieve final dilutions of $10^{-1}$ to $10^{-4}$, which were then streaked horizontally onto square trypticase soy agar plates [28]. The plates were incubated at $37{ }^{\circ} \mathrm{C}$ overnight. Controls consisted of untreated bacteria (no photosensitizers or light) kept in 96well plates for the duration of the illumination. After $18 \mathrm{~h}$ of incubation at $37{ }^{\circ} \mathrm{C}$ in the dark, CFUs were counted and the results were statistically analyzed. Survival fractions were expressed as ratios of CFUs of treated bacteria (with light and photosensitizer) to CFUs of untreated bacteria. The experiment was performed three times.

\section{Statistical analysis}

The statistical analyses have been performed using the statistical suite StatSoft (2011) Statistica (data analysis software system) version 10.0. www.statsoft.com and Excel. The quantitative variables were characterized by the arithmetic mean of standard deviation or median or maximum/ minimum (range) and $95 \%$ confidence interval. The qualitative variables were presented with the use of count and percentage. Statistical significance of differences between two groups (unpaired variables model) was processed with the Student's $t$ test. The significance of difference between more than two groups was assessed using $F$ test (ANOVA). In all the calculations the statistical significance level of $p<0.05$ has been used.

\section{Results}

Heterogeneous response of $S$. aureus to PDI

The different strains expressed a heterogeneous response to photoinactivation, ranging from 0 to $4.51 \log _{10}$-unit reductions in viable counts under the same experimental conditions. According to lethal or sub-lethal damage caused by PDI treatment, the strains were classified as sensitive or resistant PDI responders (cut-offs for the categorization presented in Table 4). Strains revealing reduction in viable counts ranged between lethal and sub-lethal damage were assigned as intermediate-sensitive (Table 4).

The SCCmec element does not have an impact on PDI response

To determine if any relationship exists between the PDI response and the type of SCCmec, SCCmec typing was performed for 321 clinical MRSA strains (for the rest of the 97 isolates SCCmec types had already been characterized). The
Table 4 Range of effectiveness of photosensitization of MRSA isolates

\begin{tabular}{llc}
\hline $\log _{10}$-unit reduction & Classification $^{\mathrm{a}}$ & Number of isolates (\%) \\
\hline $0-0.99$ & Resistant & $253(60.50)$ \\
$1-1.99$ & Intermediate-sensitive & $111(26.55)$ \\
$2-4.51$ & Sensitive & $54(12.95)$ \\
Total & & $418(100.0)$ \\
\hline
\end{tabular}

${ }^{\text {a }}$ Categorization made owing to lethal and sub-lethal damage caused by photoinactivation

results showed that for the MRSA strains studied, the distribution of PDI responders among the five different groups classified according to SCCmec types is similar (Fig. 1). The same conclusion could be drawn when comparing the distribution of uncategorized values of responses to PDI across $S$. aureus groups with different SCCmec types (Fig. 2). We can therefore conclude that the type of SCCmec element has no impact on the strain's response to PDI and could not classify strains according to their response to photodynamic treatment.

Susceptibility to photodynamic oxidation and clonal complex

Four-hundred eighteen MRSA isolates varied in their response to photodynamic inactivation. The results showed that for the MRSA strains studied, the distribution of PDI responders among the seven different groups classified according to clonal complex is different (Fig. 1). Interestingly, all CC1 $S$. aureus strains $(n=5)$ were identified as PDI-resistant isolates. Conversely, all CC30 isolates $(n=3)$ were characterized as PDI-sensitive or -intermediate-sensitive strains (Fig. 1). Also, the percentage of $\mathrm{CC} 45$ isolates increased in accordance with susceptibility to photoinactivation (Fig. 1; not statistically significant analysis). When analyzing the uncategorized values of response to PDI, clonal complex typing revealed significant differences in $S$. aureus response according to its genetic background (Fig. 3). Strains belonging to CC1 showed strict association with resistance to photoinactivation, whereas CC30 and CC8 (ST247) isolates were in general susceptible to PDI (Fig. 3). As different behavior was observed for strains CC8 according to their STs, ST number was provided in parenthesis (ST8 and ST247 respectively). Number of isolates representing each clonal complex is provided in Table 2. Results obtained indicated possible association of CC-mediated determination of genetic background and susceptibility to photodynamic oxidation.

Susceptibility to photodynamic oxidation and spa types

Determination of genetic background with spa typing also indicated its association with susceptibility to photodynamic 


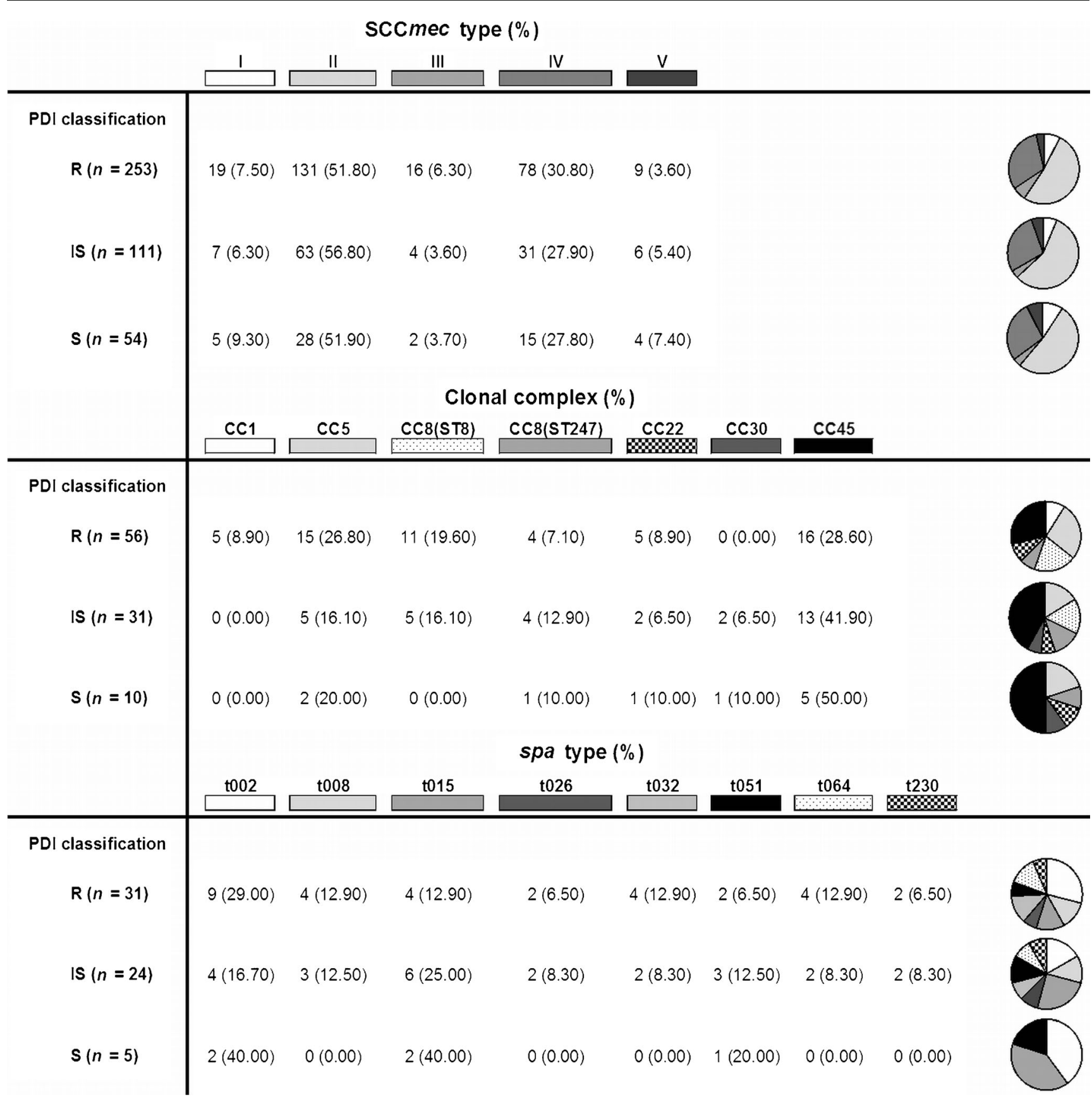

Fig. 1 Distribution of methicillin-resistant Staphylococcus aureus (MRSA) S. aureus clusters (staphylococcal cassette chromosome (SCC)mec types, clonal complexes and spa types) in photoinactivation (PDI) categories. $R$ PDI-resistant strains, $I S$ PDI-intermediate-sensitive strains, $S$ PDI-sensitive strains

inactivation as statistically different PDI responses were observed in respect of spa types (Fig. 4). Interestingly, spa typing led to distinguishing the CC45 cluster into PDIsensitive and -intermediate-sensitive strains. When analyzing solely the clonal complex, isolates belonging to CC45 generally showed intermediate sensitivity to photodynamic treatment (Fig. 3). When applying spa typing, the differentiation of PDI-sensitive strains was observed (t015) (Fig. 4). This spa type was detected exclusively within CC45 (Table 3). The analyses of distribution of PDI responders across different spa types also revealed that the percentage of 015 and t051 isolates increased in accordance with susceptibility to photoinactivation (Fig. 1). Conversely, the percentage of t064 isolates increased in accordance with resistance to PDI (Fig. 1). These observations indicated the possible association of genetic background of $S$. aureus isolates with susceptibility to photodynamic oxidation (not statistically significant analysis). 


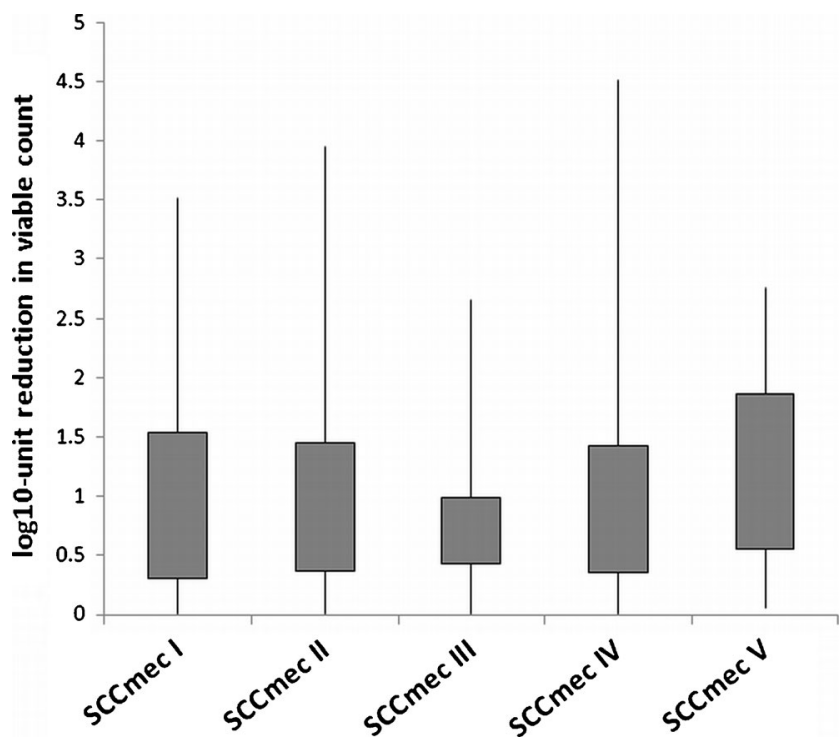

Fig. 2 Response to protoporphyrin IX (PPIX)-mediated PDI of $S$. aureus strains with various types of SCCmec element. Each box plot represents the spread of bacterial response across different clinical isolates. The error bars represent minimum and maximum values of $\log _{10}$-unit reduction in viable counts

Similar PDI response across MRSA and MSSA strains belonged to $\mathrm{CC} 22$ and $\mathrm{CC} 45$

To investigate whether the same susceptibility and/or resistance to photoinactivation could be observed in $S$. aureus isolates regardless of MRSA or MSSA status, the PDI response was analyzed in MRSA and MSSA isolates belonging to the same genetic background. Interestingly, the response of $\mathrm{CC} 22$ and CC45 S. aureus isolates was the same in the MRSA and MSSA

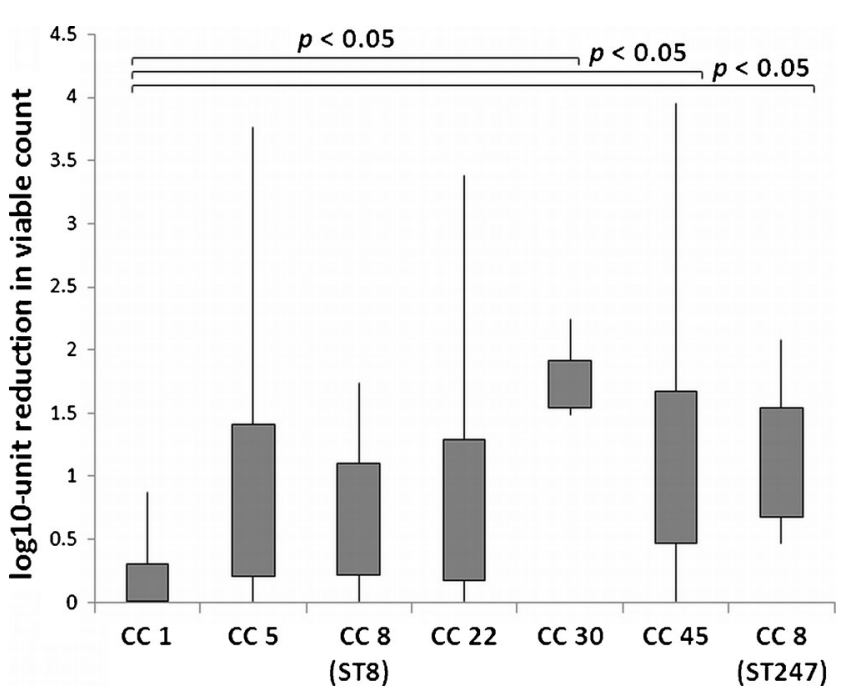

Fig. 3 Response to PPIX-mediated PDI of $S$. aureus strains from various clonal complexes. Each box plot represents the spread of bacterial response across different clinical isolates. The error bars represent minimum and maximum value of $\log _{10}$-unit reduction in viable counts

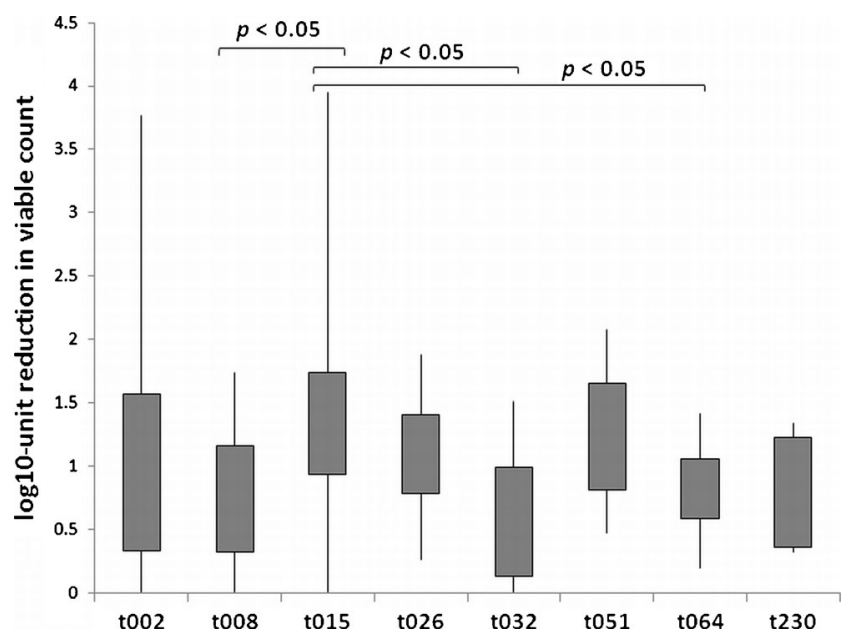

Fig. 4 Response to PPIX-mediated PDI of $S$. aureus strains with various spa types. Each box plot represents the spread of bacterial response across different clinical isolates. The error bars represent minimum and maximum value of $\log _{10}$-unit reduction in viable counts

groups indicating that the associations observed are independent of presence of SCCmec element (Fig. 5).

\section{Discussion}

Recently we demonstrated that a strain-dependent response of $S$. aureus to PDI exists, although the underlying mechanism remains unexplained [22, 23, 29]. The current study aimed to investigate statistically relevant correlations between the genetic backgrounds of methicillin-resistant $S$. aureus strains and their response to photodynamic inactivation. Thus,

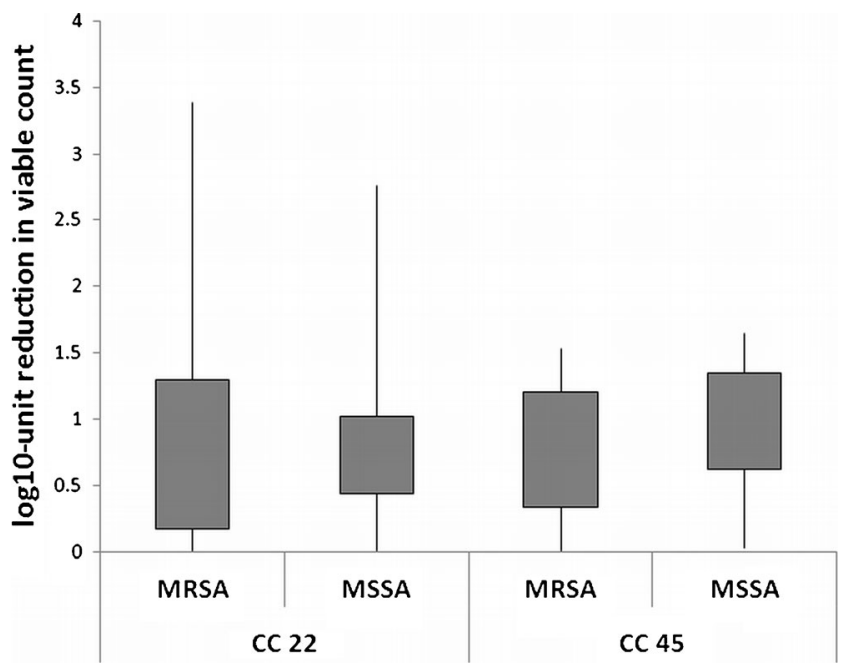

Fig. 5 Response to PPIX-mediated PDI of MRSA and MSSA strains belonging to the same clonal clusters. Each box plot represents the spread of bacterial response across different clinical isolates. The error bars represent minimum and maximum value of $\log _{10}$-unit reduction in viable counts 
evaluation of response to photodynamic treatment in distinct clonal lineages of $S$. aureus was performed.

It might be concerning that the concentration of DMSO used in the experiments could affect the results obtained. Ghajar and Harmon [30] studied the influence of DMSO on the permeability of $S$. aureus, demonstrating that DMSO increased the oxygen uptake, but reduced the rate of glycine transport. DMSO exerts a marked inhibitory effect on a wide range of bacteria and fungi. For $S$. aureus, the minimal inhibitory concentrations of DMSO vary between 20 and $30 \%$ and minimal bactericidal concentrations are above $30 \%$ [31]. $2.5 \%$ DMSO (used in the current study) is not lethal but is high enough to possibly alter membrane permeability depending on the surface protein concentration [30, 32]. To exclude the possible impact of DMSO on PPIX uptake, the intracellular concentration of DMSO-soluble PPIX and water-soluble PPIX derivative (protoporphyrin diarginate, $\mathrm{PPArg}_{2}$ ) was measured after $30 \mathrm{~min}$ incubation at $37^{\circ} \mathrm{C}$. The level of PPArg 2 uptake was measured previously [33]. The intracellular uptake of both PPIX and PPArg 2 was similar (35 vs $38 \mu \mathrm{g}$ per $\mathrm{mg}$ of bacterial protein) indicating that $2.5 \%$ DMSO did not affected the intracellular uptake of sensitizers [33]. It is worth emphasizing that photodynamic treatment including porphyrin derivatives sensitizers, could utilize DMSO concentrations of up to $25 \%$, revealing that the $2.5 \%$ DMSO used in the current study is in fact a very low dose [34]. Thus, we can conclude that the differences observed in $S$. aureus response to PPIX-mediated photoinactivation are not affected by the $2.5 \%$ DMSO used.

Previously, we have shown that MRSA strains were found to have a generally higher percentage of PDI resistance than MSSA strains [22]. This result suggested that PDI resistance may be related to multidrug-resistance in MRSA strains, thus prompting our investigation of the staphylococcal cassette chromosome mec element. The data obtained during this study allowed us to conclude that the SCCmec element has no effect on the strain response to PDI, as there were no statistically significant differences between SCCmec types and the response to PDI. Thus, we can assume that the observed phenomenon of a higher number of PDI-resistant strains within the MRSA group is a secondary effect not directly connected to antibiotic resistance. The same conclusion could be drawn when comparing the susceptibility to PDI in MRSA and MSSA groups belonging to the same clonal complexes (Fig. 5).

Different SCCmec elements may be observed in virtually identical genetic backgrounds, and conversely, strains from completely different clonal complexes may acquire the same SCCmec elements (Table 3) [35]. Thus, it was necessary to further investigate the possible association of susceptibility to PDI with clonal complexes or spa clusters. This study revealed that elevated resistance of $S$. aureus to PDI could be associated with clonal complex $\mathrm{CC} 1$ and observed in $100 \%$ of these isolates. Furthermore, clustering the isolates into clonal complexes and spa types led to identification of PDI-sensitive $S$. aureus lineages. The most PDI-sensitive isolates were characterized within CC30 and spa types t015 and t051. However, one must remember that in the current study the number of representatives in some spa types was too low to draw statistically relevant conclusions and further investigations should be performed to indicate evidence-based associations.

In summary, the present study revealed that particular genetic backgrounds could be a predisposing factor for elevated resistance to photodynamic treatment. Results of the present study corroborate the potential of $\mathrm{CC}$ typing procedure that could be applied to disclose the major lineages represented in $S$. aureus collection and to detect the PDIresistant phenotypes. These can then be further identified accurately by other powerful techniques, such as spa typing.

It is significant to underline that the resistance mechanisms toward photodynamic inactivation have not yet been observed $[18,19]$. The phenomenon that is described within the current paper concerns resistant phenotypes; thus, applying more rigorous photodynamic conditions (like a higher light dose or sensitizer concentration) this resistance could be overcome. Nevertheless, it is clinically relevant to identify isolates requiring stricter conditions to adjust the photoinactivation procedure ensuring a successful therapeutic effect. We believe that enhanced investigation concerning the association of $S$. aureus genetic background with susceptibility to photodynamic inactivation could lead to precise identification of PDI-resistant phenotypes. These studies should include more numerous groups of clonal clusters with respect to clonal complex and other spa types. Moreover, it is important to make it clear that aPDT efficacy depends on the ability of the photosensitizer and light to penetrate bacterial membranes; thus, more studies must be carried out using other types of sensitizing agents to prove the same behavior.

$S$. aureus infections continues to be a growing burden for the healthcare system. The latest epidemiological data suggest that this problem will continue to grow as the number of atrisk patients rises. Moreover, antibiotic resistance spreads from healthcare settings to the community and the development of alternative therapeutic approach is needed. One of the possibilities could be photodynamic inactivation; however, it is also not without limitations. One is the existence of PDIresistant phenotypes that require more rigorous treatment conditions to achieve the total eradication. To improve the therapeutic outcome an effort is needed in prediction of isolate response to photodynamic treatment including a better diagnostic of resistant phenotypes. The strategies to overcome PDI-resistant phenotypes as well as mechanisms leading to PDI resistance have been described elsewhere [21, 29, 36]; however, the impact of genotypic background on the susceptibility of different strains to PDI remains unexplored and 
should be a field of great interest in the future as it could have a significant clinical impact.

Acknowledgements We are grateful to Prof. Teruyo Ito from the Department of Bacteriology, School of Medicine, Department of Infection Control Science, Graduate School of Medicine, Juntendo University, Hongo Bunkyo-ku, Tokyo, Japan, for providing us with the reference strains for the SCCmec element controls. Strains of CC22 were kindly provided by Dr Rosario Rodicio University of Oviedo, Department of Functional Biology, Spain. MALDI-TOF MS analysis was performed at LM Bruss Laboratory in Poland. This work was supported by the grant no. 1651/B/P01/2010/39 from the National Science Centre (NCN). The work performed at NMI was partially supported by the Ministry of Science and Higher Education, Poland (Mikrobank 2 Programme).

Conflict of interest The authors declare that they have no conflict of interest.

Open Access This article is distributed under the terms of the Creative Commons Attribution License which permits any use, distribution, and reproduction in any medium, provided the original author(s) and the source are credited.

\section{References}

1. Kurlenda J, Grinholc M, Jasek K, Wegrzyn G (2007) RAPD typing of methicillin-resistant Staphylococcus aureus: a 7-year experience in a Polish hospital. Med Sci Monit 13:MT13-MT18

2. Josten M, Reif M, Szekat C, Al-Sabti N, Roemer T, Sparbier K, Kostrzewa M, Rohde H, Sahl HG, Bierbaum G (2013) Analysis of the matrix-assisted laser desorption ionization-time of flight mass spectrum of Staphylococcus aureus identifies mutations that allow differentiation of the main clonal lineages. J Clin Microbiol 51:1809-1817

3. Melles DC, Gorkink RF, Boelens HA, Snijders SV, Peeters JK, Moorhouse MJ, van der Spek PJ, van Leeuwen WB, Simons G, Verbrugh HA, Van BA (2004) Natural population dynamics and expansion of pathogenic clones of Staphylococcus aureus. J Clin Invest 114:1732-1740

4. Argudin MA, Mendoza MC, Vazquez F, Guerra B, Rodicio MR (2011) Molecular typing of Staphylococcus aureus bloodstream isolates from geriatric patients attending a long-term care Spanish hospital. J Med Microbiol 60:172-179

5. Katayama Y, Ito T, Hiramatsu K (2000) A new class of genetic element, staphylococcus cassette chromosome mec, encodes methicillin resistance in Staphylococcus aureus. Antimicrob Agents Chemother 44:1549-1555

6. Descloux S, Rossano A, Perreten V (2008) Characterization of new staphylococcal cassette chromosome mec ( $\mathrm{SCCmec}$ ) and topoisomerase genes in fluoroquinolone- and methicillin-resistant Staphylococcus pseudintermedius. J Clin Microbiol 46:1818-1823

7. Ito T, Ma XX, Takeuchi F, Okuma K, Yuzawa H, Hiramatsu K (2004) Novel type $\mathrm{V}$ staphylococcal cassette chromosome mec driven by a novel cassette chromosome recombinase, ccrC. Antimicrob Agents Chemother 48:2637-2651

8. Ma XX, Ito T, Tiensasitorn C, Jamklang M, Chongtrakool P, BoyleVavra S, Daum RS, Hiramatsu K (2002) Novel type of staphylococcal cassette chromosome mec identified in community-acquired methicillin-resistant Staphylococcus aureus strains. Antimicrob Agents Chemother 46:1147-1152

9. Ito T, Katayama Y, Asada K, Mori N, Tsutsumimoto K, Tiensasitorn C, Hiramatsu K (2001) Structural comparison of three types of staphylococcal cassette chromosome mec integrated in the chromosome in methicillin-resistant Staphylococcus aureus. Antimicrob Agents Chemother 45:1323-1336

10. Nastaly P, Grinholc M, Bielawski KP (2010) Molecular characteristics of community-associated methicillin-resistant Staphylococcus aureus strains for clinical medicine. Arch Microbiol 192:603-617

11. Ganga R, Riederer K, Sharma M, Fakih MG, Johnson LB, Shemes S, Khatib R (2009) Role of SCCmec type in outcome of Staphylococcus aureus bacteremia in a single medical center. J Clin Microbiol 47: 590-595

12. Stefani S, Chung DR, Lindsay JA, Friedrich AW, Kearns AM, Westh H, Mackenzie FM (2012) Meticillin-resistant Staphylococcus aureus (MRSA): global epidemiology and harmonisation of typing methods. Int J Antimicrob Agents 39:273-282

13. Hallin M, Friedrich AW, Struelens MJ (2009) Spa typing for epidemiological surveillance of Staphylococcus aureus. Methods Mol Biol 551:189-202

14. Kharkwal GB, Sharma SK, Huang YY, Dai T, Hamblin MR (2011) Photodynamic therapy for infections: clinical applications. Lasers Surg Med 43:755-767

15. St Denis TG, Dai T, Izikson L, Astrakas C, Anderson RR, Hamblin MR, Tegos GP (2011) All you need is light: antimicrobial photoinactivation as an evolving and emerging discovery strategy against infectious disease. Virulence 2:509-520

16. Wainwright M (1998) Photodynamic antimicrobial chemotherapy (PACT). J Antimicrob Chemother 42:13-28

17. Tavares A, Dias SR, Carvalho CM, Faustino MA, Tome JP, Neves MG, Tome AC, Cavaleiro JA, Cunha A, Gomes NC, Alves E, Almeida A (2011) Mechanisms of photodynamic inactivation of a gram-negative recombinant bioluminescent bacterium by cationic porphyrins. Photochem Photobiol Sci 10:1659-1669

18. Pedigo LA, Gibbs AJ, Scott RJ, Street CN (2009) Absence of bacterial resistance following repeat exposure to photodynamic therapy. Proc SPIE 7380

19. Jori G, Coppellotti O (2007) Inactivation of pathogenic microorganisms by photodynamic techniques: mechanistic aspects and perspective applications. Anti-Infect Agents Med Chem 6:119-131

20. Nakonieczna J, Rapacka-Zdonczyk A, Kawiak A, Bielawshi KP, Grinholc M (2013) Sub-lethal photodynamic inactivation renders Staphylococcus aureus susceptible to silver nanoparticles. Photochem Photobiol Sci 12:1622-1627

21. Grinholc M, Richter M, Nakonieczna J, Fila G, Bielawski KP (2011) The connection between agr and SCCmec elements of Staphylococcus aureus strains and their response to photodynamic inactivation. Photomed Laser Surg 29:413-419

22. Grinholc M, Szramka B, Kurlenda J, Graczyk A, Bielawski KP (2008) Bactericidal effect of photodynamic inactivation against methicillin-resistant and methicillin-susceptible Staphylococcus aureus is strain-dependent. J Photochem Photobiol B 90:57-63

23. Grinholc M, Zawacka-Pankau J, Gwizdek-Wisniewska A, Bielawski KP (2010) Evaluation of the role of the pharmacological inhibition of Staphylococcus aureus multidrug resistance pumps and the variable levels of the uptake of the sensitizer in the strain-dependent response of Staphylococcus aureus to PPArg(2)-based photodynamic inactivation. Photochem Photobiol 86:1118-1126

24. Nakonieczna J, Grinholc M (2012) Photodynamic inactivation requires innovative approach concerning numerous bacterial isolates and multicomponent sensitizing agents. Photodiagnosis Photodyn Ther 9:359-361

25. CLSI (2007) Performance standards for antimicrobial susceptibility testing. CLSI approved standard M100-S17. Clinical and Laboratory Standards Institute, Wayne

26. Szabados F, Woloszyn J, Richter C, Kaase M, Gatermann S (2010) Identification of molecularly defined Staphylococcus aureus strains using matrix-assisted laser desorption/ionization time of flight mass spectrometry and the Biotyper 2.0 database. J Med Microbiol 59: 787-790 
27. Ghaznavi-Rad E, Nor SM, Sekawi Z, Van BA, Neela V (2010) A simplified multiplex PCR assay for fast and easy discrimination of globally distributed staphylococcal cassette chromosome mec types in meticillin-resistant Staphylococcus aureus. J Med Microbiol 59: $1135-1139$

28. Jett BD, Hatter KL, Huycke MM, Gilmore MS (1997) Simplified agar plate method for quantifying viable bacteria. Biotechniques 23:648650

29. Nakonieczna J, Michta E, Rybicka M, Grinholc M, GwizdekWisniewska A, Bielawski KP (2010) Superoxide dismutase is upregulated in Staphylococcus aureus following protoporphyrinmediated photodynamic inactivation and does not directly influence the response to photodynamic treatment. BMC Microbiol 10:323

30. Ghajar BM, Harmon SA (1968) The effect of dimethyl sulfoxide (DMSO) on permeability of Staphylococcus aureus. Biochem Biophys Res Commun 32:940-944

31. Basch H, Gadebusch HH (1968) In vitro antimicrobial activity of dimethylsulfoxide. Appl Microbiol 16:1953-1954

32. Gurtovenko AA, Anwar J (2007) Modulating the structure and properties of cell membranes: the molecular mechanism of action of dimethyl sulfoxide. J Phys Chem B 111:10453-10460

33. Kossakowska M, Nakonieczna J, Kawiak A, Kurlenda J, Bielawski KP, Grinholc M (2013) Discovering the mechanisms of straindependent response of Staphylococcus aureus to photoinactivation: oxidative stress toleration, endogenous porphyrin level and strain's virulence. Photodiagnosis Photodyn Ther DOI:10.1016/j.pdpdt. 2013.02.004

34. Lambrechts SA, Demidova TN, Aalders MC, Hasan T, Hamblin MR (2005) Photodynamic therapy for Staphylococcus aureus infected burn wounds in mice. Photochem Photobiol Sci 4: 503-509

35. Monecke S, Coombs G, Shore AC, Coleman DC, Akpaka P, Borg M, Chow H, Ip M, Jatzwauk L, Jonas D, Kadlec K, Kearns A, Laurent F, O'Brien FG, Pearson J, Ruppelt A, Schwarz S, Scicluna E, Slickers P, Tan HL, Weber S, Ehricht R (2011) A field guide to pandemic, epidemic and sporadic clones of methicillin-resistant Staphylococcus aureus. PLoS One 6:e17936

36. Vera DM, Haynes MH, Ball AR, Dai T, Astrakas C, Kelso MJ, Hamblin MR, Tegos GP (2012) Strategies to potentiate antimicrobial photoinactivation by overcoming resistant phenotypes. Photochem Photobiol 88:499-511

\section{Author's contribution}

MG conceived and designed the experiments, analyzed the data, and wrote the paper; MG and AR-Z performed the experiments; MG, JE, ARL, AP contributed reagents/materials/analysis tools.

All authors read and approved the final manuscript. 